\title{
Prevention of contrast nephropathy after cardiac catheterisation
}

Increasing numbers of patients in the developed world are exposed to contrast medium during cardiac catheterisation procedures, and the pressure to increase cardiac laboratory throughput of patients with suspected coronary disease is unrelenting. For this reason, the problem of radiocontrast induced nephropathy (RCIN) assumes greater and greater importance. How is the fallout from contrast exposure to be minimised?

\section{Scale of the problem}

Radiocontrast use in all branches of medicine is reported to be the third most common cause of new onset renal failure in hospital patients. ${ }^{1}$ Although varying definitions of nephropathy have been employed in the literature, including a $25 \%$ rise, a $50 \%$ rise or a doubling of serum creatinine concentration, it is clear that this is a common complication of cardiac catheterisation. In the recent epidemiological report by McCullough and colleagues, 1826 unselected patients undergoing coronary intervention were found to have a $14.5 \%$ incidence of acute contrast induced renal failure as defined by a $25 \%$ rise in serum creatinine. ${ }^{2}$ This may seem a relatively small decline in renal function but it has important consequences in terms of prolonged in-patient stay and cost. ${ }^{3}$ More severe derangements in renal function increase morbidity, mortality, and the risk of developing end stage renal failure. ${ }^{34}$ Of the McCullough group, 7.7 per 1000 required renal replacement therapy, an outcome that was associated with a $36 \%$ in-hospital mortality and a two year survival of less than $20 \%$. The incidence of RCIN is likely to be higher following interventional procedures than after angiography alone but is certainly not confined to the former.

\section{Patients at highest risk}

The pathogenesis of RCIN is likely complex. ${ }^{5}$ Currently, it is widely accepted that ischaemic damage to the renal medulla and a direct toxic effect of contrast medium upon renal tubular cells is of importance. Patients most at risk are those with impaired renal function as judged by an increase in serum creatinine concentration, a risk that appears to rise exponentially as creatinine increases. ${ }^{6}$ The presence of diabetes further amplifies this risk leading to an approximate doubling of incidence. ${ }^{78}$ There is no convincing evidence, however, that diabetic patients with normal renal function are particularly vulnerable. ${ }^{8}$

External factors which increase risk include the volume and concentration of contrast medium employed ${ }^{9}$ (none of the patients receiving less than $100 \mathrm{ml}$ of contrast in the McCullough study developed acute renal failure requiring blood purification), blood volume depletion at the time of contrast exposure (usually avoidable), use of high osmolality rather than low osmolality contrast medium in patients with pre-existing renal impairment, ${ }^{10}$ and perhaps drugs such as angiotensin converting enzyme (ACE) inhibitors and non-steroidal inflammatory agents. Increasing age and heart failure have been cited as factors in the development of nephropathy. ${ }^{11}{ }^{12}$ However, the evidence that these are independent risk factors is inconclusive and may reflect diuretic use and relative blood volume depletion in those with heart failure and an overestimate of renal function from the serum creatinine in elderly patients with a low muscle mass.

\section{Natural history}

The typical patient with RCIN is non-oliguric. The serum creatinine peaks $4-5$ days after contrast exposure and a creatinine estimated at 72 hours will detect $90 \%$ of those affected. It remains unclear what proportion of patients recover completely though certainly some sustain permanent renal damage. A minority become dialysis dependent. Patients undergoing coronary angiography often have vascular disease elsewhere and the differential diagnosis includes atheromatous embolisation which carries a worse prognosis. The conditions may of course coexist.

\section{Trials of prevention}

Prevention trials have focused on the use of blood volume expansion, low osmolality versus high osmolality contrast, dopamine, calcium channel blockers, mannitol, diuretic treatment, atrial natriuretic peptide, ACE inhibitors, the adenosine antagonist theophylline, endothelin receptor antagonists, and, most recently, acetylcysteine. Animal experiments have, in general, given contradictory results and their applicability to the human situation is doubtful. Convincing evidence of benefit following coronary angiography in humans with impaired renal function has been observed only with the use of $0.45 \%$ (half normal) saline ${ }^{3}$ and low osmolality contrast medium. ${ }^{13}$ Tepel and colleagues have recently reported benefit from the oral administration of the antioxidant acetylcysteine in such patients exposed to intravenous contrast. ${ }^{14}$ However, the number studied (83) was small as was the dose of contrast used $(75 \mathrm{ml})$ and no patient in the treated or control group required dialysis.

\section{A scheme for prevention}

Patients undergoing cardiac catheterisation and contrast exposure fall naturally into two main groups: non-urgent and urgent. A common example of the former is the patient with stable angina pectoris to be booked for day case coronary angiography. The latter group includes those with unstable angina and acute myocardial infarction.

The scheme we propose is based upon incomplete evidence and is our best estimate of a reasonable approach. Running through these recommendations are a limited number of basic rules that we feel should be applied to all patients visiting the angiography laboratory: (1) the serum creatinine should be known; (2) those with renal impairment (serum creatinine above the upper limit of normal for the laboratory concerned or known reduction in 
glomerular filtration rate) should receive pre-hydration and low osmolality contrast medium; (3) contrast exposure should be avoided in volume depleted patients; and (4) the minimum dose of contrast consistent with diagnostic angiographic quality should be used and rapid re-exposure avoided where possible.

\section{NON-URGENT PATIENTS}

In a non-diabetic patient with no history of renal disease, it is reasonable to classify the patient as low risk if creatinine concentration has been documented as within the normal range during the previous year. Care should be taken in interpreting the serum creatinine in elderly patients and if doubt exists the patient should be classified as high risk. ACE inhibitors, non-steroidal anti-inflammatory agents, and diuretics should be stopped a few days before the investigation if possible. Account should be taken of special circumstances. For example, the Royal College of Radiologists now recommend that metformin be withheld for 48 hours after the procedure and reinstituted only after renal function has been re-evaluated and found to be equivalent to the baseline value. If the patient has a renal transplant in situ and femoral access is to be used, it is probably wise to plan to employ the femoral artery contralateral to the graft.

Fluid deprivation for more than four hours preprocedure should be avoided and if delay occurs or hypovolaemia is suspected clinically there should be a low threshold for administration of intravenous normal $(0.9 \%)$ or half normal $(0.45 \%)$ saline. These measures are appropriate in patients with a normal serum creatinine concentration.

In patients with evidence of renal impairment we do not recommend day case investigation. The patient should receive 1 litre of saline in the 12 hours before and a further 1 litre in the 12 hours after the examination, and should be encouraged to drink if thirsty except for the four hours pre-procedure. In all patients with pre-existing renal dysfunction serum creatinine concentration should be checked one and four days post-procedure and the results should be made known to the responsible cardiologist. ${ }^{15} \mathrm{In}$ the UK this will usually require input from the patient's family doctor.

We recommend either normal or half normal saline despite the fact that trials showing benefit have employed $0.45 \%$ saline. The latter is seldom used in the UK and we doubt whether there is any special advantage in its use. We also advocate pre-procedure admission to hospital in patients at higher risk despite claims that a hydration programme at home may be successful. ${ }^{16}$ Further study of such programmes is required. Fears that cost precludes pre-admission of high risk patients seem misplaced given the costs associated with contrast nephropathy if it occurs. Finally we advocate 12 hours of intravenous saline treatment before contrast exposure but emphasise that no clarity exist as to the ideal duration of such treatment precontrast. Randomised, prospective controlled studies are badly needed in this area.

\section{URGENT PATIENTS}

Frequently serum creatinine concentration will be known in such patients, as they will have been referred for urgent investigation from within the hospital environment. Those in whom serum creatinine concentration is normal can be regarded as at low risk of developing contrast nephropathy and can be managed as outlined above. Those who appear volume depleted and those in whom serum creatinine concentration is elevated require intravenous fluid and we suggest that normal saline be commenced before or at the time of contrast exposure. A reasonable regimen would be $500 \mathrm{ml}$ saline over half an hour followed by 1 litre in the succeeding 12 hours providing pulmonary oedema is absent. Again, serum creatinine concentration should be checked one day and four days after contrast exposure.

We emphasise that the above scheme is our estimate of a reasonable approach based upon currently available information but that large gaps exist in our knowledge of the optimum approach to prevention of this common problem. Future research is needed. Greater awareness of the problem will do much to reduce its impact.

Department of Cardiology,

C S R BAKER

London Chest Hospital,

Bonner Road,

London E2 9BJ, UK

cbaker6751@aol.com

L R I BAKER

Renal Unit,

St Bartholemew's Hospital,

West Smithfield,

London EC1A $7 B E$, UK

1 D'Elia JA, Gleason RE, Alday M, et al. Nephrotoxicity from angiographic contrast material. A prospective study. Am F Med 1982;72:719-25.

2 McCullough PA, Wolyn R, Rocher LL, et al. Acute renal failure after coronary intervention: incidence, risk factors, and relationship to mortality. $A m \mathcal{F}$ Med 1997;103:368-75.

3 Solomon R, Werner C, Mann D, et al. Effects of saline, mannitol, and furosemide to prevent acute decreases in renal function induced by radiocontrast agents. $N$ Engl f Med 1994;331:1416-20.

4 Levy EM, Viscoli CM, Horwitz RI. The effect of acute renal failure on mortality. A cohort analysis. $\mathcal{F} A M A$ 1996;275:1489-94.

5 Solomon R. Contrast-medium-induced acute renal failure. Kidney Int 1998; 53:230-42.

6 Davidson CJ, Hlatky M, Morris KG, et al. Cardiovascular and renal toxicity of a nonionic radiographic contrast agent after cardiac catheterization. A prospective trial. Ann Intern Med 1989;110:119-24.

7 Barrett BJ. Contrast nephrotoxicity. F Am Soc Nephrol 1994;5:125-37.

8 Parfrey PS, Griffiths SM, Barrett BJ, et al. Contrast material-induced renal failure in patients with diabetes mellitus, renal insufficiency, or both. A prospective controlled study. N Engl f Med 1989;320:143-9.

9 Albert SG, Shapiro MJ, Brown WW, et al. Analysis of radiocontrast-induced nephropathy by dual-labeled radionuclide clearance. Invest Radiol 1994;29: 618-23.

10 Barrett BJ, Carlisle EJ. Meta-analysis of the relative nephrotoxicity of highand low-osmolality iodinated contrast media. Radiology 1993;188:171-8.

11 Rich MW, Crecelius CA. Incidence, risk factors, and clinical course of acute renal insufficiency after cardiac catheterization in patients 70 years of age or older. A prospective study. Arch Intern Med 1990;150:1237-42.

12 Taliercio CP, Vlietstra RE, Fisher LD, et al. Risks for renal dysfunction with cardiac angiography. Ann Intern Med 1986;104:501-4.

13 Rudnick MR, Goldfarb S, Wexler L, et al. Nephrotoxicity of ionic and nonionic contrast media in 1196 patients: a randomized trial. The iohexol cooperative study. Kidney Int 1995;47:254-61.

14 Tepel M, van der GM, Schwarzfeld C, et al. Prevention of radiographiccontrast-agent-induced reductions in renal function by acetylcysteine. $N$ Engl f Med 2000;343:180-4.

15 Porter GA. Experimental contrast-associated nephropathy and its clinical implications. Am f Cardiol 1990;66:18F-22F

16 Taylor AJ, Hotchkiss D, Morse RW, et al. PREPARED: preparation for angiography in renal dysfunction: a randomized trial of inpatient vs outpatient hydration protocols for cardiac catheterization in mild-to-moderate renal dysfunction. Chest 1998;114:1570-4. 